\title{
Avaliação automática de habilidades sensório-motoras em simulador odontológico
}

\author{
Lucas H. Sallaberry \\ Interlab - EPUSP \\ Universidade de São Paulo - USP \\ São Paulo, Brasil \\ lucas.sallaberry@usp.br
}

\author{
Romero Tori \\ Interlab - EPUSP \\ Universidade de São Paulo - USP \\ São Paulo, Brasil \\ tori@usp.br
}

\author{
Fatima L. S. Nunes \\ LApIS - EACH/USP e Interlab - EPUSP \\ Universidade de São Paulo - USP \\ São Paulo, Brasil \\ fatima.nunes@usp.br
}

\begin{abstract}
Resumo-Simuladores em realidade virtual voltados para a saúde possibilitam o treinamento de estudantes antes do primeiro contato com o paciente, de maneira segura e sem gasto de material. Dados coletados durante a execução do treinamento favorecem a implementação de sistemas de avaliação automática, que podem tanto fornecer retorno de desempenho para o aluno durante o treino, sem a necessidade de um instrutor presente, quanto auxiliar o professor durante avaliações. Estudos que utilizam aprendizado de máquina para realizar a avaliação de aprendizes em simuladores de realidade virtual com retorno háptico ainda são escassos na literatura. Este trabalho propõe a utilização de técnicas de aprendizado de máquina para definir um método de avaliação a fim de mensurar a aquisição de habilidades sensório-motoras de estudantes. $O$ modelo será validado em um simulador de anestesia odontológica em realidade virtual. Dados de especialistas e alunos iniciantes e experientes foram coletados durante experimentos e serão utilizados para treinar o modelo. Possíveis métricas de desempenho e técnicas de avaliação foram levantadas em uma revisão sistemática da literatura.
\end{abstract}

Index Terms-Avaliação de aprendiz, simulador odontológico, retorno háptico, aprendizado de máquina.

\section{INTRODUÇÃO}

Simuladores em realidade virtual (RV) focados no ensino e treinamento na área da saúde possibilitam realizar, com segurança, treinamentos que se aproximam de situações reais, além de viabilizar a automatização de avaliações [1]-[3] e a realização do mesmo treinamento múltiplas vezes sem consumo de material, entre outros benefícios proporcionados pela tecnologia [4].

O VIDA Odonto é um simulador de procedimentos odontológicos em RV e com retorno de força. Desenvolvido em uma parceria do Laboratório de Tecnologias Interativas (Interlab - EPUSP), Laboratório de Aplicações de Informática em Saúde (LApIS - EACH/USP) e Laboratório de Simulação e Treinamento (LaSiT - FOB/USP), o foco atual do sistema é o treinamento da aplicação de anestesias de maneira realista e confiável, especificamente para o bloqueio do nervo alveolar inferior, sendo que novos procedimentos serão implementados futuramente [5].

\section{MotivaÇÃo}

Simuladores em RV com retorno de força possibilitam a coleta de diversos dados relacionados às ações do aprendiz de maneira automática, sem a necessidade de periféricos adicionais. Estes dados podem ser utilizados para a implementação de sistemas de avaliação de habilidades, capazes de fornecer retorno de desempenho do treinamento para o aluno automaticamente, oferecendo maior autonomia ao seu aprendizado. Estes sistemas também podem beneficiar o professor, auxiliandoo na avaliação de estudantes por meio de informações baseadas em dados objetivos (obtidos com o dispositivo háptico). Tais informações muitas vezes são difíceis de examinar com exatidão por observação visual, tal como velocidade, força e precisão nos movimentos.

\section{Trabalhos Correlatos}

Em um levantamento realizado na forma de revisão sistemática da literatura, foram encontrados estudos em simuladores abrangendo variados tipos de procedimentos médicos que apresentavam avaliação de aprendizes. Considerando que cada estudo poderia apresentar mais de um tipo de método de avaliação, técnicas estatísticas, tal como abordado por Anjos et al. [1], foram predominantes entre os estudos e utilizadas em 86,5\% deles. Trabalhos como Esen et al. [3], que utilizam medidas de desempenho para calcular um valor final correspondente ao nível de habilidade do aprendiz (chamado nota ou score), compuseram 36,5\% dos trabalhos levantados. Apenas um estudo utilizou técnica de aprendizado de máquina [2], indicando que ainda há muito a ser explorado em questão de aplicações deste tipo de técnica na área.

Dias et al. [6] sugerem um crescente número de estudos utilizando aprendizado de máquina para avaliar competência em procedimentos médicos nos últimos anos, porém a maioria deles ainda está em estágio inicial e necessita de uma validação mais rigorosa.

\section{Solução Proposta}

A proposta deste projeto é o desenvolvimento de um modelo capaz de avaliar aprendizes automaticamente a partir de dados objetivos coletados em simuladores de RV com retorno de força. Como prova de conceito, será utilizado o simulador VIDA Odonto, um sistema de treinamento de anestesia odontológica em RV [5]. Dados de especialistas (dentistas e professores) e de alunos de odontologia experientes e inexperientes serão utilizados para treinar algoritmos de 
aprendizado de máquina a fim de torná-los capazes de avaliar novos aprendizes.

A Figura 1 mostra a arquitetura do modelo proposto. Os dados coletados consistem na posição e orientação da seringa ao longo do tempo. A partir deles, os erros dos dispositivos de rastreio serão analisados para que se possa eliminar eventuais ruídos e, então, serão calculadas métricas referentes ao desempenho do participante. Uma etapa de extração e seleção de características poderá também ser realizada caso as técnicas escolhidas não realizem este processo automaticamente e o vetor de características resultante será utilizado para treinar e testar o algoritmo. Após treinado, pretende-se integrar o modelo ao simulador odontológico. Novas coletas de dados poderão ser realizadas em experimentos ou em sala de aula e o aluno receberá, em tempo real, o resultado de sua avaliação.

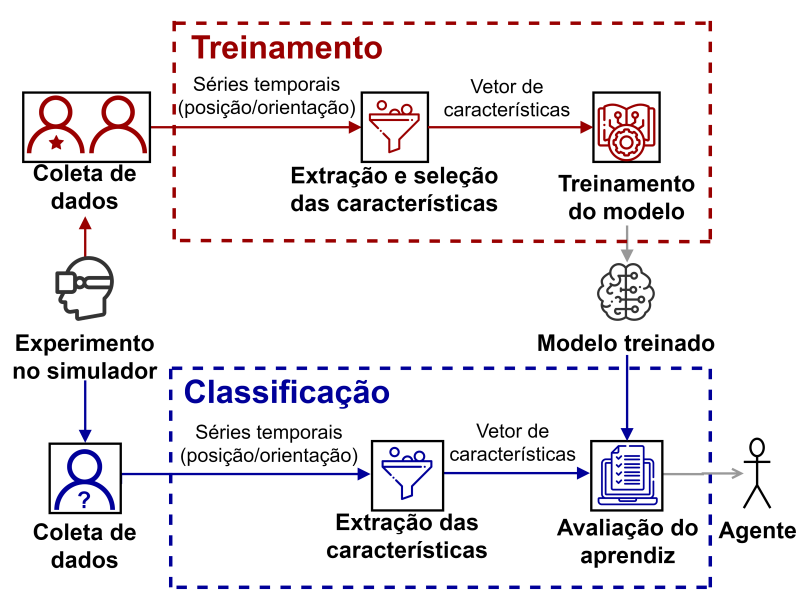

Figura 1. Arquitetura do modelo proposto.

A capacidade de um simulador conseguir diferenciar um usuário novato de um experiente é conhecida na literatura como validade de constructo [7]. Tomando como premissa que usuários experientes são mais habilidosos que usuários novatos, um algoritmo capaz de classificar aprendizes entre novatos e experientes é capaz de avaliar a habilidade sensóriomotora de um usuário indiretamente.

\section{Resultados Preliminares}

Uma revisão sistemática da literatura foi conduzida para identificar métricas e técnicas utilizadas para avaliar automaticamente as habilidades sensório-motoras de aprendizes em simuladores com retorno háptico voltados para a saúde. Diversos estudos reportaram sucesso em diferenciar o desempenho de novatos e experientes. O tempo total para realização do procedimento foi a métrica de desempenho mais utilizada nos estudos $(63,5 \%$ de uso). A distância total percorrida pelo instrumento durante o procedimento e a distância do instrumento em relação a um alvo pré determinado também foram métricas muito utilizadas $(28,8 \%$ e $15,4 \%$ respectivamente).

Algumas das principais técnicas de aprendizado de máquina já foram estudadas, tal como redes neurais, random forests, máquinas de vetores de suporte, entre outras, porém pretendese buscar mais algoritmos que se adéquem ao problema. Como os dados brutos coletados se tratam de dados temporais, será também estudada a viabilidade de algoritmos que utilizem este tipo de informação para classificação.

Dados de 16 especialistas (dentistas e professores), 51 estudantes de odontologia sem experiência e 193 estudantes com experiência no procedimento de anestesia do nervo alveolar inferior foram coletados em experimentos. Como técnicas de aprendizado de máquina geralmente exigem grande número de instâncias para treinamento, novos experimentos podem ser necessários. Alguns métodos capazes de trabalhar com um número reduzido de amostras, tal como interactive machine learning, também serão explorados. Em trabalhos anteriores, um modelo baseado na técnica $T^{2}$ de Hotelling e utilizando dados de 163 desses participantes foi capaz de classificar corretamente os estudantes entre aprovados e não aprovados, baseado nas avaliações de um professor especialista, com acurácia de $84,0 \%$ [8], porém ainda não foi realizado um estudo utilizando os dados de todos os participantes e comparando-os com base no nível de experiência.

\section{CONClus Ão}

Há vários trabalhos na literatura que buscam avaliar aprendizes em procedimentos médicos de forma objetiva, porém a aplicação de técnicas de aprendizado de máquina para este fim ainda foi pouco investigada e validada.

Ao final deste trabalho, espera-se desenvolver um modelo de avaliação objetiva de habilidades sensório-motoras que possa ser integrado ao simulador VIDA Odonto e validado com novos aprendizes.

\section{AgRadecimentos}

A Figura 1 foi produzida com recursos criados por Freepik e disponíveis em Flaticon.com.

\section{REFERÊNCIAS}

[1] A. M. Anjos, R. Tori, A. Castro, S. Oliveira, and F. L. S. Nunes, "Statistical methods in the evaluation of sensory-motor skills acquisition in 3D interactive virtual environments," in Proceedings of the 29th Annual ACM Symposium on Applied Computing, ser. SAC '14, Gyeongju, Republic of Korea, 2014, pp. 223-228.

[2] P. Rhienmora, P. Haddawy, S. Suebnukarn, and M. N. Dailey, "Intelligent dental training simulator with objective skill assessment and feedback," Artificial Intelligence in Medicine, vol. 52, no. 2, pp. 115-121, Jun. 2011.

[3] H. Esen, A. Sachsenhauser, K. Yano, and M. Buss, "A Multi-User Virtual Training System Concept and Objective Assessment of Trainings," in RO-MAN 2007 - The 16th IEEE International Symposium on Robot and Human Interactive Communication, Jeju, South Korea, 2007, pp. 10841089.

[4] C. G. Correa, R. Tori, and F. L. Nunes, "Haptic Simulation for Virtual Training in Application of Dental Anesthesia," in 2013 XV Symposium on Virtual and Augmented Reality, Cuiabá - Mato Grosso, Brazil, May 2013, pp. 63-72.

[5] R. Tori, G. Z. Wang, L. H. Sallaberry, A. A. Tori, E. C. Oliveira, and M. A. A. M. Machado, "VIDA ODONTO: Ambiente de Realidade Virtual para Treinamento Odontológico," Revista Brasileira de Informática na Educação, vol. 26, no. 02, pp. 80-101, May 2018.

[6] R. D. Dias, A. Gupta, and S. J. Yule, "Using Machine Learning to Assess Physician Competence: A Systematic Review," Academic Medicine, vol. 94, no. 3, pp. 427-439, Mar. 2019.

[7] E. M. McDougall, "Validation of Surgical Simulators," Journal of Endourology, vol. 21, no. 3, pp. 244-247, Mar. 2007.

[8] E. Collaço, "Impact of immersion on the performance of haptic virtual reality for inferior alveolar nerve anesthesia training." Doutorado em Sistemas Digitais, Universidade de São Paulo, São Paulo, 2019. 